\title{
Deep Embedding Features for Salient Object Detection
}

\author{
Yunzhi Zhuge, ${ }^{\dagger}$ Yu Zeng, ${ }^{\dagger}$ Huchuan Lu $^{\dagger}$ \\ ${ }^{\dagger}$ Dalian University of Technology \\ $\dagger\{$ zgyz, zengyu $\} @$ mail.dlut.edu.cn, ${ }^{\dagger}$ lhchuan@ dlut.edu.cn
}

\begin{abstract}
Benefiting from the rapid development of Convolutional Neural Networks (CNNs), some salient object detection methods have achieved remarkable results by utilizing multi-level convolutional features. However, the saliency training datasets is of limited scale due to the high cost of pixel-level labeling, which leads to a limited generalization of the trained model on new scenarios during testing. Besides, some FCN-based methods directly integrate multi-level features, ignoring the fact that the noise in some features are harmful to saliency detection. In this paper, we propose a novel approach that transforms prior information into an embedding space to select attentive features and filter out outliers for salient object detection. Our network firstly generates a coarse prediction map through an encorder-decorder structure. Then a Feature Embedding Network (FEN) is trained to embed each pixel of the coarse map into a metric space, which incorporates much attentive features that highlight salient regions and suppress the response of non-salient regions. Further, the embedded features are refined through a deep-to-shallow Recursive Feature Integration Network (RFIN) to improve the details of prediction maps. Moreover, to alleviate the blurred boundaries, we propose a Guided Filter Refinement Network (GFRN) to jointly optimize the predicted results and the learnable guidance maps. Extensive experiments on five benchmark datasets demonstrate that our method outperforms state-of-the-art results. Our proposed method is end-to-end and achieves a realtime speed of 38 FPS.
\end{abstract}

\section{Introduction}

Salient object detection, which aims to estimate the visual significance of image regions, has arisen widely discussions in recent years. It serves as a pre-processing step for many computer vision tasks, such as image regartigating (Fang et al. 2012), image classification (Schmid, Jurie, and Sharma 2012) and quality assessment (Zhang, Shen, and Li 2014). However, due to many uncertain factors such as cluttered backgrounds and complex scenarios, it still remains a difficult task.

Earlier saliency detection methods (Li et al. 2013) (Jiang et al. 2013) generate saliency maps under the guidance of heuristic priors(e.g. color, texture and contrast). However,

Copyright (C) 2019, Association for the Advancement of Artificial Intelligence (www.aaai.org). All rights reserved. these low-level features can hardly capture high-level semantic relations of the objects and its surroundings. Thus the low-level based methods are not robust enough to distinguish salient objects from cluttered background.

Recently, deep convolutional neural networks have shown outstanding performance in many recognition tasks. Owning to its hierarchical structure, CNNs can learn multi-level features from training samples. Compared with the handcrafted features, the CNNs features are more semantically rich. Therefore, the CNNs based saliency detection methods (Wang et al. 2016; Liu and Han 2016) have achieved impressive results by leveraging high-level semantic features to capture foreground areas. However, the CNNs based methods still have two apparent deficiencies. On the one hand, downsampling operations such as pooling and convolution dramatically reduce the resolution of initial image, which degrade the details such as image boundary. On the other hand, many CNNs based methods (Zhang et al. 2017a; Wang et al. 2017b; Zhang et al. 2018a) introduce overloaded layers to integrate multi-level features. Such excessive processes often cause features cluttered, and thus cause the incorrect saliency detection results.

To resolve the afore-mentioned problems, in this paper, we propose a novel multi-branch model to leverage deep embedding features (DEF) for salient object detection. The DEF network is composed of four components: a backbone for Initial Saliency Prediction (ISP), Feature Embedding Network (FEN), Recursive Feature Integration Network (RFIN) and Guided Filter Refinement Network (GFRN). ISP is a lightweight module that generates prior saliency maps and multi-scale side output features as a preprocessing step. FEN embeds the prior saliency maps and the features into a metric space to weight the spatial importance of each element in feature maps. FEN can filter unrelated information in the side output features and thus generate better features that focus on salient regions. To remedy the downsampling information loss, we propose RFIN to integrate embedded features with residual reconstructed features in a deep-to-shallow manner. In each level of RFIN, a stage-wise saliency map is predicted and supervised by the ground-truth to enhance the features robustness. The saliency map of each stage also serves as a guidance for the next stage. Finally, we propose GFRN to further enhance the boundary consistency and smoothness of the last 


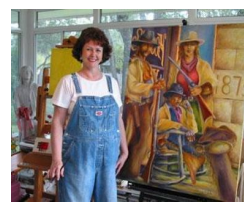

(a)

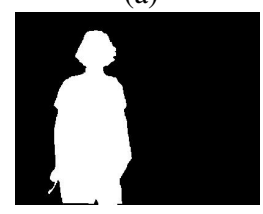

(d)

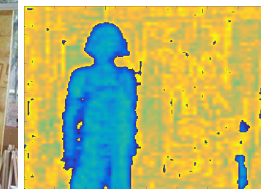

(b)

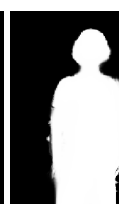

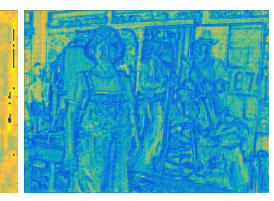

(c)

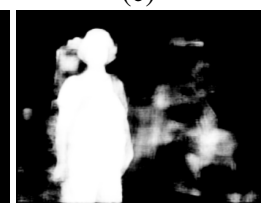

(f)
Figure 1: Visual comparison with a multi-level feature based method. From top-left to bottom-right: (a) Input image. (b)(c) Feature maps of our method and Amulet (Zhang et al. 2017a). (d) Ground Truth. (e)-(f) Saliency maps of our method and Amulet.

saliency maps predicted by RFIN. GFRN transforms the original RGB image into a guidance map to refine boundaries of the salient objects. As is shown in Figure 1, compared with an existing method Amulet (Zhang et al. 2017a) based on multi-level features integration, our method can generate more precise saliency map in the guidance of robust feature maps.

In summary, each component in our algorithm plays a role in enhance the accuracy for salient object detection. Our main contributions are as follows:

- Motivated by the aforementioned drawbacks of directly integrating multi-level features, we suggest to boost salient object detection results in a new point, i.e., embedding prior predictions into a metric space to filter outliers and generate attentive features to precisely localize salient objects.

- We propose a Recursive Feature Integration Network (RFIN) which progressively refines the embedding features by integrating them with multi-level features in a deep-to-shallow sequence.

- The proposed method achieves state-of-the-art results on five large-scale benchmark datasets. It also achieves a real-time speed of 38 FPS using one 1080 Ti GPU.

\section{Related Work}

Over the past decades, a large set of saliency detection methods have been developed. Saliency detection methods can be roughly divided into two categories: methods based on hand-crafted features and deep learning based methods.

\section{Methods Based on Hand-crafted Features}

Earlier saliency detection methods mainly focused on exploiting hand-crafted features, which can be categorized as local and global schemes. Local methods measure localcontrast to evaluate saliency. In (Schölkopf, Platt, and Hofmann 2006), the equilibrium distribution over map locations are treated as activation and saliency values. In contrast,

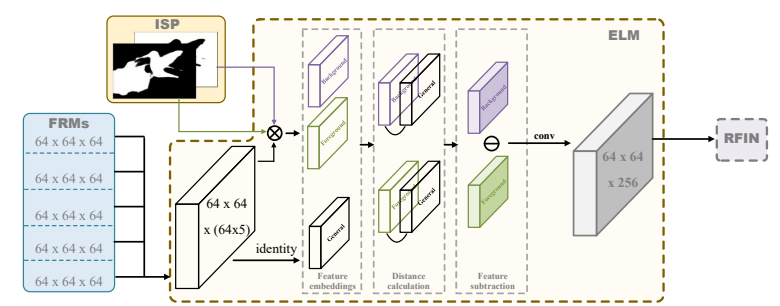

Figure 2: The structure of Feature Embedding Network.

global methods considering both color statistics and holistic contrast of the whole image. In (Liu et al. 2011), conditional random field is learned to effectively combine local and global features for saliency detection. (Jiang et al. 2013) propose a method to map the regional feature vector to a saliency score using Random Forest regressor. (Cheng et al. 2011) utilize color-discriminative features and global contrast to obtain optimal saliency maps.

\section{Deep Learning Based Methods}

Recently, deep convolutional neural networks (CNNs) have delivered superior performance in many recognition tasks (Krizhevsky, Sutskever, and Hinton 2012; He et al. 2016). In earlier research (Wang et al. 2015; Li and Yu 2015; Zhao et al. 2015), image patches are the basic processing unit for saliency prediction. Although these algorithms perform superior than hand-crafted feature based methods, they are relatively sparse in spatial and time-consuming due to the fully connected layers. To resolve this problem, several attempts based on fully convolutional networks (FCNs) are proposed. (Liu and Han 2016) propose a deep hierarchical network that progressively refine saliency maps by integrating local context information. (Wang et al. 2016) take predicted saliency maps as input to recurrently refine the generated saliency maps by rectify its previous errors. In (Hou et al. 2017), short connections are proposed to convert high-level features into shallower layers, which locate salient regions and refine the sparse details simultaneously. (Zhang et al. 2017a) integrate and combine multi-level feature maps to simultaneously incorporate coarse semantics and fine details. (Wang et al. 2017b) propose a stage-wise refinement model with a pyramid pooling to extract global context information and local details for salient object detection. (Zhang et al. 2018b) firstly denote that some features are redundant for salient object detection. An attention guided network is proposed to selectively integrates multilevel contextual information and alleviate distraction of cluttered features. Different from the above feature integration based methods, we propose a multi-branch deep embedding feature network which can simultaneously map coarse prediction map into a metric space and incorporate multiple side output features recursively to exploits both global and local information.

\section{Feature Embedding}

Embedding Features has been used in saliency detection and semantic segmentation. (Li et al. 2015a) embeds low-level 


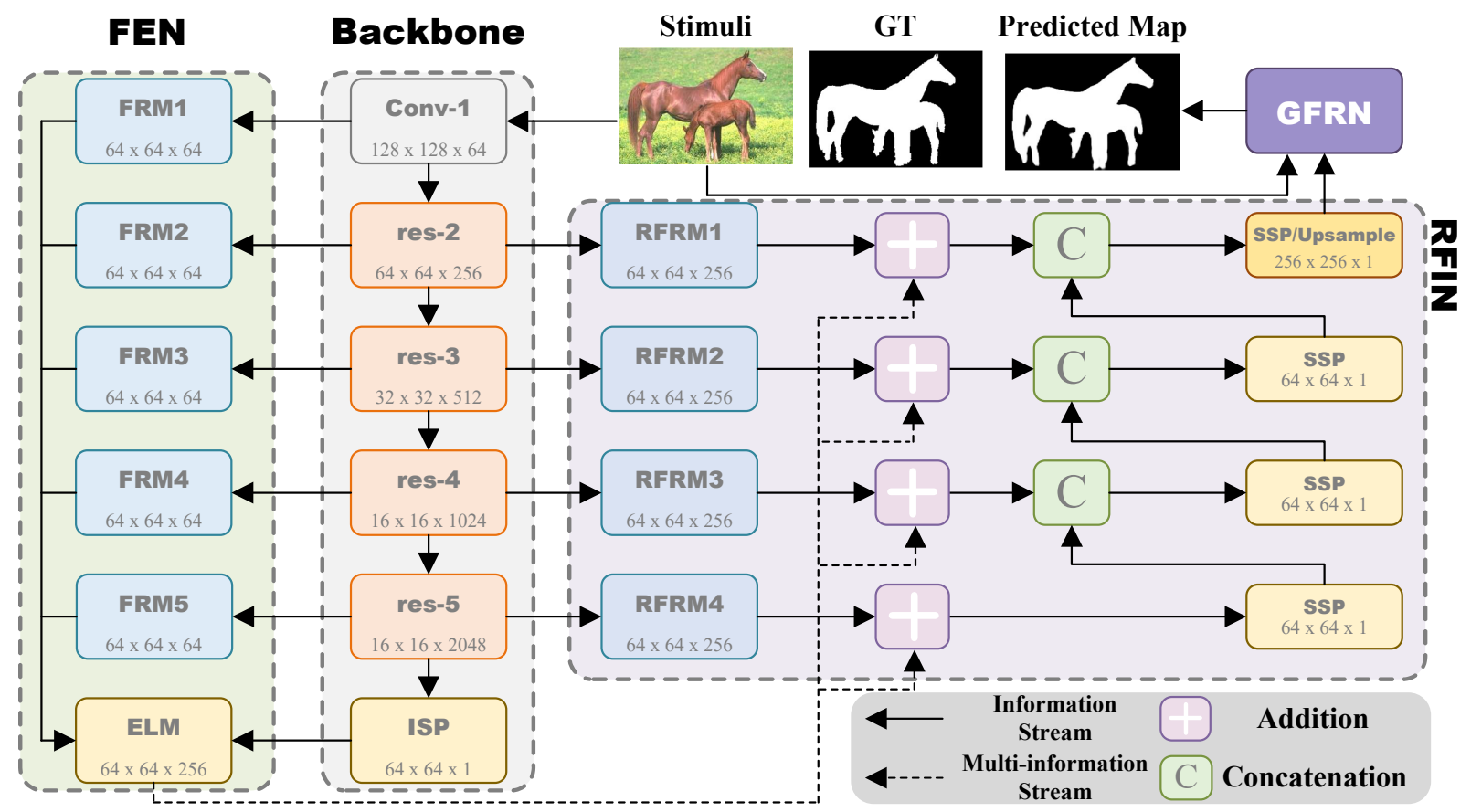

Figure 3: The pipeline of our method. Each module or block is presented by a solid box. FEN-Feature Embedding Network. RFIN-Recursive Feature Integration Network. GFRN-Guided Filter Refinement Network. ISP-Initial Saliency Prediction. SSP-Stagewise Saliency Prediction. FRM-Feature Reshape Module. RFRM-Residual Feature Reshape Module. ELM-Embedding Learning Module.

features into high-level features, which leverages the advantage of CNNs to simultaneously capture semantic information in high-level features as well as spatial and contrast information in low-level features. In (Liu et al. 2018), a fully convolutional network is trained to learn the feature embedding space for each superpixel. The learned feature embedding corresponds to a similarity measure between two adjacent superpixels. In (Zeng et al. 2018), an image-specific classifier is learned from the attributes of training data to classify the pixels of each image.

Different from the above embedding methods, our deep feature embedding network transforms both the prior predictions and multi-level features into a metric space. With the help of the prior information about the salient regions, the generated attentive features can effectively hightlight the salient regions and suppress the backgrounds.

\section{Proposed Method}

We describe the proposed method in this section. To begin with, we describe the components of our proposed DEF architecture in the first subsection. Then we explain the detailed training schemes of our algorithms in the last subsection.

\section{Architecture}

The main architecture of our proposed algorithm is shown in Figure 3. It is a multi-branch network consisting of four components: Initial Saliency Network (ISN), Deep Feature Embedding Network (FEN), Recursive Feature Integration
Network (RFIN) and Guided Filter Refinement Network (GFRN). ISN provides the initial saliency predictions and multi-scale side output features. FEN embeds the predictions and features of ISN into a metric space to weight the spatial importance of each element in feature maps. RFIN integrates embedded features with residual reconstructed features and predicts a series of stage-wise saliency maps in deep-to-shallow manner. GFRN takes original RGB image and the last saliency map produced by RFIN as inputs. The RGB image is transformed into a guidance map to refine boundaries of the salient objects.

Initial Saliency Network We format our Initial Saliency Network on the basis of fully convolutional network. We choose ResNet101 (He et al. 2016) as our backbone due to its fast convergence characteristic and astonishing results in image classification task. ResNet101 is composed of five basic blocks with different output dimensions: conv1, res2, res3, res4, and res5. The output spatial of basic blocks are decreased by a stride of 2 . To obtain larger feature maps, we set the stride of the last block to 1 . For computation efficiency, we choose the output of last two blocks as side output layers, denoted as Side $_{4}$ and Side $_{5}$. To reduce dimensions, we pass them through two convolution layers with $256 \mathrm{ker}-$ nels. The initial prediction map are produced by feeding the two feature maps into a convolution layer with 1 kernels and upsampled to $64 \times 64$.

Feature Embedding Network After obtaining the initial prediction maps and multi-level feature maps, we use the 


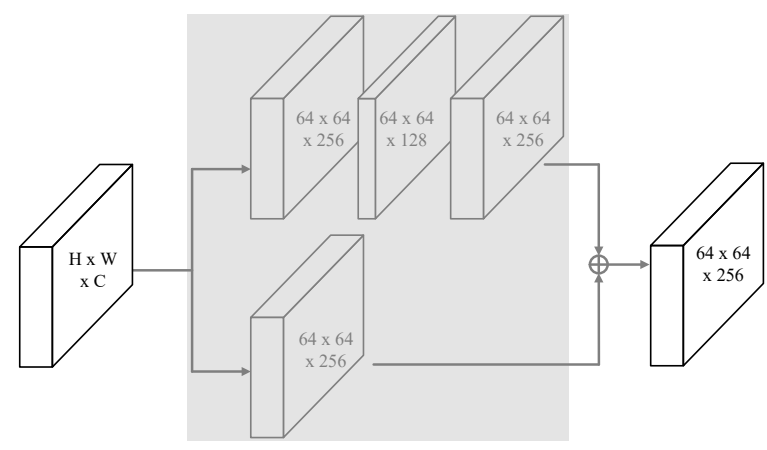

Figure 4: Overall architecture of Residual Feature Reshape Module.

Feature Embedding Network (FEN) to map the features and initial prediction maps into a metric space. FEN is composed of paralleled Feature Reshape Modules (FRM) and Embedding Learning Module (ELM), As is shown in Figure 2. To begin with, we utilize Feature Reshape Modules to integrate multi-level feature maps. Features of different spatial resolutions are resized into the same scale through pooling or upsampling operations. Then we use a convolutional layer with 64 kernels to reduce dimensions. Let $\mathrm{Side}_{l}$ denotes feature maps of level $l(l=1,2, \ldots, 5)$. All feature maps are resized to the same spatial size of $64 \times 64$. The integrated feature maps are generated by

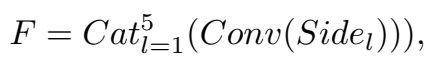

in which Conv and Cat denotes convolution and concatenation operation respectively. The resulting integrated feature map is of the shape $64 \times 64 \times 320$, where $64 \times 64$ represents spatial size and 320 represents number of channels.

Denote $x^{m}$ as a pixel of image $X$ in position $m$. Given an initial saliency map $S_{1}$, we first obtain a reverse saliency map $S_{0}$ by $S_{0}=1-S_{1}$ that highlights background area. Then, each pixel of saliency and reverse saliency maps is mapped into a 320-dimensional vector by

$$
\boldsymbol{\varphi}_{m k}=\boldsymbol{\mu}\left(s_{m k} ; \boldsymbol{\psi}\right), k=0,1,
$$

where $s_{m k}$ is the value of $S_{k}$ in position $m . \boldsymbol{\varphi}_{m 1}$ and $\boldsymbol{\varphi}_{m 0}$ are the embedding vector of the pixel in position $m$ of saliency map and the reverse saliency map, respectively. $\boldsymbol{\mu}$ and $\psi$ represents embedding operation and its parameter.

Given an image $I$, we obtain the attentive features of the pixel in position $m$ according to:

$$
V\left(I_{m}\right)=\left|\operatorname{Dis}\left(\boldsymbol{\varphi}_{m 1}, \mathbf{f}_{m}\right)-\operatorname{Dis}\left(\boldsymbol{\varphi}_{m 0}, \mathbf{f}_{m}\right)\right|,
$$

where $\mathbf{f}_{\mathbf{m}}$ denotes vector of integrated feature maps in position $m$. Dis $(\cdot, \cdot)$ denotes Euclidean distance.

Recursive Feature Integration Network The Feature Embedding Network effectively embeds saliency maps into metric space to generate attentive features. Though we can directly apply a softmax layer to obtain relatively precise prediction saliency maps, some detailed areas are still ignored. Therefore, we propose a Recursive Feature Integration Network to supplement detailed information.

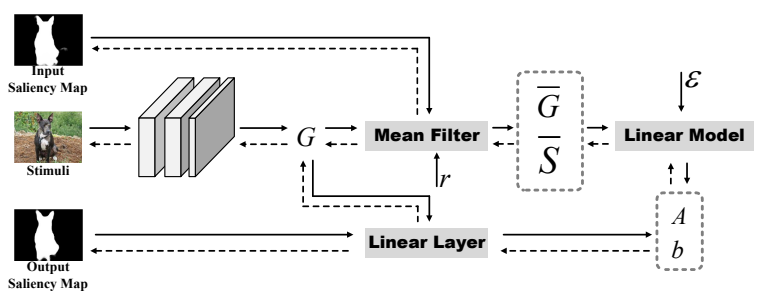

Figure 5: Computation process of Guided Filter Refinement Network. GFRN takes original image and saliency map generated by last step as inputs, generating refined saliency map with sharp boundary. $\longrightarrow$ represents forward stream and $-\rightarrow$ represents back propagation.

As is shown in Figure 3, Recursive Feature Integration Network takes embedded feature maps Em and multi-scale features as input. To begin with, Residual Feature Reshape Module (RFRM) is proposed to reconstruct side-output features to facilitate it for further integration (depicted in Figure 4). Assume that the spatial size of input features is $\left(R^{H \times W \times C}\right)$, we utilize two paralleled convolution layers to reshape features and learn complementary information for integration. In each step, RFIN learns to supplement details of embedded feature maps and rectify prediction results of the last step. The stage-wise saliency predictions of step $i$ can be produced by:

$$
\mathbf{P}^{\mathbf{i}}=\left\{\begin{array}{l}
W_{i} * C a t\left(\left(\mathbf{E m}+\mathbf{R F R}^{l}\right), \mathbf{P}^{\mathbf{i}-\mathbf{1}}\right)+b, i=2,3,4 \\
W_{i} *\left(\mathbf{E m}+\mathbf{R} \mathbf{F} \mathbf{R}^{l}\right)+b, i=1
\end{array}\right.
$$

where $*$ and $W_{i}$ represents convolution operation and its parameters to generate prediction maps. $\mathbf{R F R}^{\mathbf{l}}$ represents the feature maps generated by RFIN at level $l . b$ is the bias parameter. In Equation 4, The stagewise prediction results in level $i(i=2,3,4)$ is obtained by integrating corresponding RFIN features and $i-1$ prediction maps.

Guided Filter Refinement Network The RFIN can generate finer results by recursively integrate embedded maps and multi-level features. However, due to the downsampling operation of base network, there still exists a gap between prediction results and ground truth, especially on object boundaries. To further refine details and make the boundary clear of salient objects, we adopt a Guided Filter Refinement Network (GFRN) (Wu et al. 2018) to overcome the bondage of the base network.

The computation process of GFRN is shown in Figure 5. The original RGB image and the saliency map generated by RFIN are the input of GFRN. Given a pair of inputs, convolutional layers are first added as a transformation function to change the dimension of input image, which serves as a flexible and trainable guidance map. After that, GFRN computes $A$ and $b$ by minimizing the reconstruction error between $G$ and $S$ with mean filter and linear model. Output saliency map $(O)$ is computed by an linear transformation taking $A$, $b$ and $G$ as inputs:

$$
O=A * G+b
$$




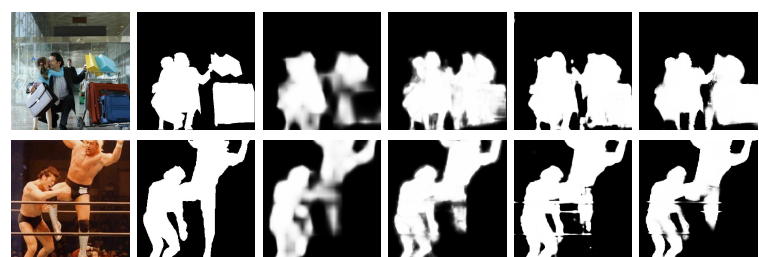

(a)

(b)

(c) (e)

(f)

Figure 6: Visual comparison of several components. From left to right: (a)Image, (b)Ground Truth, (c)Baseline, (d)FEN, (e)RFIN(stage-4), (f)GFRN.

$r$ and $\varepsilon$ represents radius of mean filter and the regularization term respectively.

\section{Training Schemes}

Our proposed multi-branch model is trained end-to-end. Input images are resized to $256 \times 256$ to match the size requirements of base network. During training, initial saliency maps generated by Initial Saliency Network are inaccurate, which effect the performance of our algorithm. To get rid of this deficiency, during training we randomly disarray the pixel of ground truth with a certain probability and serves it as input to the Feature Embedding Network (FEN). To train the model, we minimize the cross-entropy loss between each stage-wise prediction of RFIN and the ground-truth, as well as the cross-entropy loss between the final output and the groud-truth.

\section{Experiments}

To verify the effectiveness of our proposed algorithm, we conduct experiments on five public datasets (ECSSD, PASCAL-S, DUT-OMRON, DUTS and HKU-IS). We evaluate the proposed algorithm using precision-recall curves (PR-curves), mean F-measure, mean absolute error (MAE). In addition, we briefly explain the implementation details of our method and evaluate the performance of our method by comparing with other state-of-the-arts algorithms.

\section{Implementation details}

We implement our approach in Python with the Pytorch toolbox. We run our approach on a PC with a $3.7 \mathrm{GHz} \mathrm{CPU}$, 32GB RAM and a GTX 1080 Ti GPU (with $11 \mathrm{G}$ memory).

We train our model using DUTS training dataset (Wang et al. 2017b). To avoid over-fitting, we augment the training set by 4 times through horizontal flipping and vertical flipping. We use SGD to optimize our network with the momentum parameter of 0.9 and the weight decay of 0.001 . We set the base learning rate to $1 \mathrm{e}-7$ and iteration number to $30 \mathrm{~K}$. It takes around 7 hours to train our model with a mini-batch of 10 . When testing, the proposed algorithm takes around 0.026 second (38FPS) to process an image with $256 \times 256$ resolution.

\section{Datasets and Evaluation Metrics}

To evaluate the performance of our proposed methods, we adopt five benchmark datasets as follows.

ECSSD (Yan et al. 2013) is composed of 1000 images with multiple objects of different scales. This dataset contains many semantically meaningful and complex structures contends.

PASCAL-S (Li et al. 2014) is derived from PASCAL VOC2010 segmentation dataset (Everingham 2008) and contains 850 natural images.

HKU-IS (Li and Yu 2015) includes 4447 images with fined pixel-wise annotations. Images of this dataset are well chosen to include multiple disconnected salient objects or objects touching the image boundary.

DUTS (Wang et al. 2017a) is a large dataset which is composed of 10553 training images and 5019 test images with accurate pixel-wise annotations. All training images are picked out from the ImageNet DET training/val sets, while test images are picked out from the ImageNet DET test set and the SUN dataset.

DUT-OMRON (Yang et al. 2013) has a total of 5168 high-quality images. These images are chosen from more than 140,000 natural images, each of which contains one or more salient objects and relatively complex backgrounds. Thus this dataset is more difficult and challenging, and provides more space of improvement for related research in saliency detection.

Evaluation Metrics. We evaluate the performance of different salient object detection algorithms through three main metrics, including the precision-recall curves (PR curves), F-measure, mean absolute error(MAE). PR curves can be computed by binarizing the saliency map with a threshold in $[0,255]$ and then comparing the binary maps with the ground truth. To be specific, precision represents the percentage of salient pixels being correctly detected, while recall corresponds to the ratio between properly detected salient pixels and salient pixels in ground truth. In many occasions, both precision and recall are important measure metrics. Therefore F-measure, which is calculated by precision and recall, is used as an overall performance measure,

$$
F_{\beta}=\frac{\left(1+\beta^{2}\right) \times \text { Precision } \times \text { Recall }}{\beta^{2} \times \text { Precision }+ \text { Recall }} .
$$

We set $\beta^{2}$ to 0.3 to emphasize the precision. And MAE score can be calculated by average pixel-wise absolute difference between the binary ground truth and the saliency map:

$$
T=\frac{1}{W \times H} \sum_{x=1}^{W} \sum_{y=1}^{H}|S(x, y)-G(x, y)|,
$$

where $\mathrm{W}$ and $\mathrm{H}$ denote width and height of an image, $S(x, y)$ is the saliency value of the pixel at $(x, y)$.

\section{Comparison with State-of-the-arts}

We compare our algorithm with other 14 state-of-theart ones, including 12 deep learning based algorithms (BPN (Wang et al. 2018), SRM (Wang et al. 2017b), Amulet (Zhang et al. 2017a), UCF (Zhang et al. 2017b), DHS (Liu and Han 2016), NLDF (Luo et al. 2017), RFCN (Wang et al. 2016), DS (Li et al. 2015b), DLC (Li and Yu 2016), ELD (Lee, Tai, and Kim 2016), LEGS (Wang et 

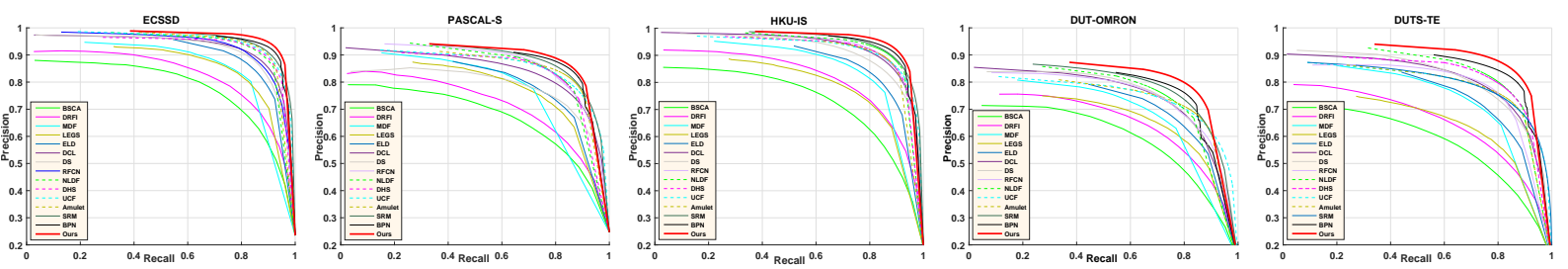

Figure 7: Precision-Recall curves of our method and other state-of-art methods on five benchmark datasets.

Table 1: The mean F-measure (larger is better) and MAE (smaller is better) of different saliency detection methods on five saliency detection datasets. The best three results are shown in bold, italic, and underlined. Our method ranks first on most of these datasets and metrics.

\begin{tabular}{|c|c|c|c|c|c|c|c|c|c|c|}
\hline \multirow{2}{*}{ Method } & \multicolumn{2}{|c|}{ ECSSD } & \multicolumn{2}{c|}{ PASCAL-S } & \multicolumn{2}{c|}{ HKU-IS } & \multicolumn{2}{c|}{ DUT-OMRON } & \multicolumn{2}{c|}{ DUTS-TE } \\
\cline { 2 - 9 } & $\mathrm{F}_{\beta}$ & $\mathrm{MAE}$ & $\mathrm{F}_{\beta}$ & $\mathrm{MAE}$ & $\mathrm{F}_{\beta}$ & $\mathrm{MAE}$ & $\mathrm{F}_{\beta}$ & MAE & $\mathrm{F}_{\beta}$ & MAE \\
\hline Ours & $\mathbf{0 . 9 1 5}$ & $\mathbf{0 . 0 3 6}$ & $\mathbf{0 . 8 2 6}$ & $\mathbf{0 . 0 7 0}$ & $\mathbf{0 . 9 0 7}$ & $\mathbf{0 . 0 3 3}$ & $\mathbf{0 . 7 6 9}$ & $\mathbf{0 . 0 6 2}$ & $\mathbf{0 . 8 2 1}$ & $\mathbf{0 . 0 4 5}$ \\
BPN (Wang et al. 2018) & 0.903 & 0.045 & 0.816 & 0.074 & 0.882 & 0.038 & 0.708 & 0.063 & 0.763 & 0.052 \\
SRM (Wang et al. 2017b) & $\underline{0.892}$ & $\underline{0.056}$ & $\underline{0.821}$ & $\underline{0.085}$ & $\underline{0.874}$ & $\underline{0.046}$ & $\underline{0.707}$ & $\underline{0.069}$ & $\underline{0.757}$ & $\underline{0.059}$ \\
Amulet (Zhang et al. 2017a) & 0.867 & 0.059 & 0.763 & 0.100 & 0.839 & 0.052 & 0.648 & 0.098 & 0.676 & 0.085 \\
UCF (Zhang et al. 2017b) & 0.841 & 0.080 & 0.701 & 0.127 & 0.808 & 0.074 & 0.613 & 0.132 & 0.629 & 0.117 \\
DHS (Liu and Han 2016) & 0.871 & 0.063 & 0.773 & 0.095 & 0.852 & 0.054 & - & - & 0.724 & 0.067 \\
NLDF (Luo et al. 2017) & 0.878 & 0.063 & 0.814 & 0.099 & 0.873 & 0.048 & 0.683 & 0.079 & 0.743 & 0.065 \\
RFCN (Wang et al. 2016) & 0.834 & 0.109 & 0.751 & 0.133 & 0.835 & 0.089 & 0.627 & 0.111 & 0.712 & 0.067 \\
DS (Li et al. 2015b) & 0.826 & 0.124 & 0.659 & 0.176 & 0.785 & 0.078 & 0.603 & 0.120 & 0.632 & 0.091 \\
DCL (Li and Yu 2015) & 0.805 & 0.108 & 0.709 & 0.146 & - & - & 0.644 & 0.092 & 0.673 & 0.101 \\
ELD (Lee, Tai, and Kim 2016) & 0.810 & 0.082 & 0.718 & 0.123 & 0.769 & 0.074 & 0.611 & 0.092 & 0.628 & 0.093 \\
LEGS (Wang et al. 2015) & 0.785 & 0.118 & 0.699 & 0.158 & 0.723 & 0.119 & 0.591 & 0.133 & 0.585 & 0.138 \\
MDF (Li et al. 2015b) & 0.807 & 0.105 & 0.709 & 0.146 & 0.801 & 0.089 & 0.644 & 0.092 & 0.673 & 0.094 \\
DRFI (Jiang et al. 2013) & 0.733 & 0.164 & 0.618 & 0.206 & 0.722 & 0.144 & 0.550 & 0.139 & 0.541 & 0.175 \\
BSCA (Qin et al. 2015) & 0.705 & 0.185 & 0.601 & 0.223 & 0.654 & 0.175 & 0.509 & 0.190 & 0.499 & 0.197 \\
\hline
\end{tabular}

al. 2015), MDF ( $\mathrm{Li}$ and Yu 2015)) and two conventional algorithms (DRFI (Jiang et al. 2013), BSCA(Qin et al. 2015)). For fair comparison, we compute other saliency maps with their original implementation details or use them provided by the authors.

Quantitative Evaluation We compare the proposed method with the others in terms of PR curves, F-measure scores and MAE scores. Figure 7 shows the proposed approach performs favorably against all the other methods. MAE scores and F-measure scores are given in Table 1. As we can see, our approach generates the best score across all datasets, which means that our method have a good perceive of salient region and can generate accurate saliency maps close to the ground truth masks.

Visual Comparison Figure 8 shows the visual comparisons of our approach and other methods. In the shown examples, we accurately segment the salient objects against multiple interference, including rare pattern (row 1), low contrast (row 2\&row 5), high-level semantic information (row 4) and complex background (row 6). From these comparisons, we can see that our method can generate more accurate saliency maps with purity backgrounds.

\section{Ablation Studies}

The proposed framework is composed of four components, including ISN, FEN, RFIN and GFRN. To show the effectiveness of each component, we take a series experiments on ECSSD and DUTS-TE datasets as follows. We take Fmeasure and MAE scores as evaluating indicators.

Effectiveness of Each Components We set the results of ISN as our baseline version. To demonstrate the effectiveness of Feature Embedding Network, we add a 1 dimension convolution layer to predict saliency maps based on the embedding features, denoted as FEN. The result of FEN shown in row 3 of Table 2 demonstrates that deep embedding features can effectively enhance the localization and detection capability of the saliency detector. As is shown in Figure 3, Recursive Feature Integration Network (RFIN) totally generates 4 stage-wise saliency predictions in one pass. We analyze the effectiveness of Recursive Feature Integration by comparison these stage-wise results. For notation simplicity, we denote $L(L=1,2,3,4)$ stage results as RFIN (stage- $L$ ). Comparison results are shown in rows 4-7 of Table 2 . Through recursively feature integration, our algorithm can produce more and more accurate results, which demonstrate that by recursively integrate the side-output features and stage-wise predictions, RFIN can effectively reduce the error of last stage.The 8 row of Table 2 indicates that Guided Filter Refinement Network can still increase the F-measure score by $1 \%$. Visual comparison of several components are shown in Figure 6

Different Backbones To demonstrate that our proposed method is flexible and can easily adapted to other framework, we extend experiments by replacing Resnet-101 


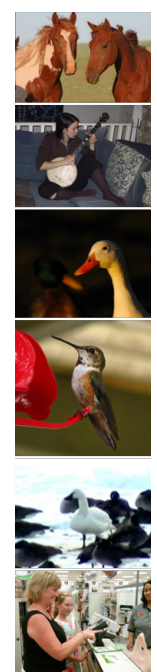

Image

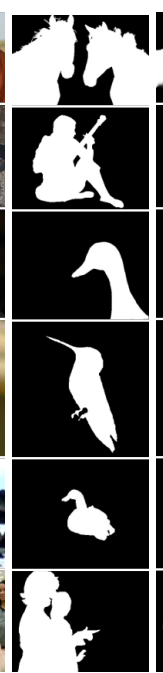

GT

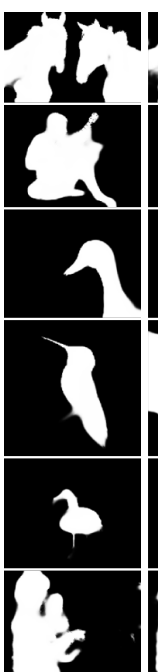

DEF

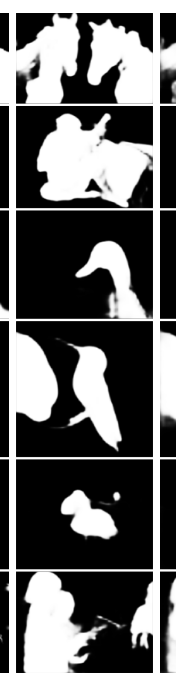

BPN

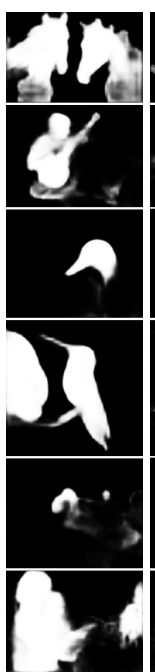

SRM

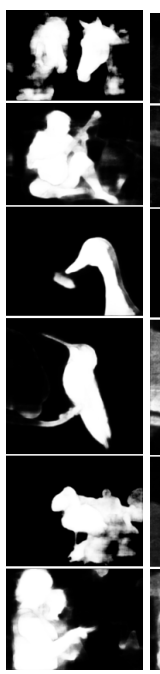

Amulet

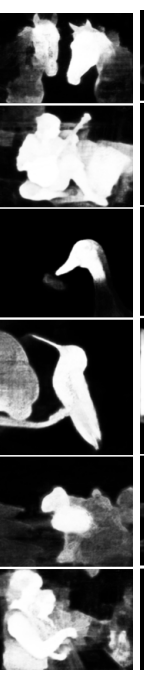

$\mathrm{UCF}$

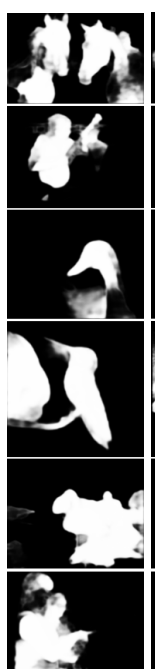

DHS

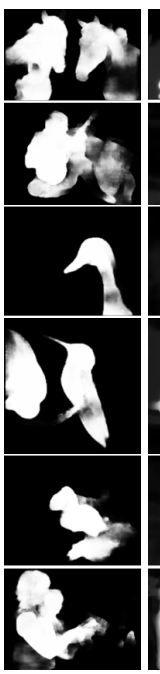

NLDF

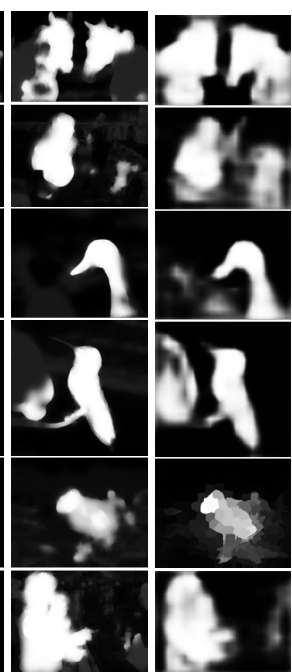

RFCN

DS

Figure 8: Visual comparison of our method and other methods. It is clear that our methods generates more accurate saliency maps than others.

Table 2: Quantitative comparison of different architectures. Baseline denotes the Initial Saliency Prediction. "FEN" in row 3 represents the direct prediction results of Feature Embedding Network. 4-7 rows represent the results of different stage-wise saliency predictions, which monotonically increase. "GFRN", denotes the prediction results of Gated Feature Refinement Network, which is our final version. The last three rows are comparisons between different backbones.

\begin{tabular}{c|cc|cc}
\hline \multirow{2}{*}{ Network Structure } & \multicolumn{2}{|c|}{ ECSSD } & \multicolumn{2}{c}{ DUTS-TE } \\
\cline { 2 - 5 } & $F_{\beta}$ & $M A E$ & $F_{\beta}$ & $M A E$ \\
\hline Baseline & 0.821 & 0.055 & 0.721 & 0.076 \\
FEN & 0.874 & 0.046 & 0.763 & 0.067 \\
RFIN(stage-1) & 0.885 & 0.044 & 0.785 & 0.061 \\
RFIN(stage-2) & 0.887 & 0.043 & 0.788 & 0.060 \\
RFIN(stage-3) & 0.892 & 0.041 & 0.794 & 0.055 \\
RFIN(stage-4) & 0.905 & 0.037 & 0.813 & 0.046 \\
\hline GFRN & $\mathbf{0 . 9 1 5}$ & $\mathbf{0 . 0 3 6}$ & $\mathbf{0 . 8 2 1}$ & $\mathbf{0 . 0 4 5}$ \\
DRFN-V & 0.889 & 0.043 & 0.774 & 0.056 \\
GRFN-D & 0.891 & 0.041 & 0.792 & 0.053 \\
\hline
\end{tabular}

with two other networks as backbone, i.e. VGG-16 and DenseNet-161. For VGG-16 version, we take 5 feature maps to constitute multi-level features in our work, which are conv1-2, conv2-2, conv3-3, conv4-3 and conv5-3, respectively. For DenseNet-161 version, we extract features in the last layer of each denseblock, we denote them as dense 1 , dense 2 , dense 3 and dense 4 . We keep other settings unchanged to control variable. The results of both versions are shown in Table 2. From comparing the results of last three rows, we can observe that the proposed method works well for different backbones.

\section{Conclusion}

In this paper, we propose a novel deep embedding feature network for salient object detection. Different from existing methods which directly fused multi-level features to generate the prediction maps, we put forward an embedding learning architecture to embed initial saliency map into feature vectors and recursively narrow the gap between stage-wise predictions and ground truth. A Convolutional Guided Filter is also utilized to strengthen overall performance. Extensive evaluations demonstrate that our approach achieves the state-of-the-art results. Except for the salient object detection, our DEF possesses the potential of dealing with other low-level vision tasks.

\section{Acknowledgement}

This work was supported by the Natural Science Foundation of China under Grant 61725202, 61751212, 61771088, 61632006 and 91538201.

\section{References}

Cheng, M. M.; Mitra, N. J.; Huang, X.; Torr, P. H. S.; and Hu, S. M. 2011. Global contrast based salient region detection. In Proc. of IEEE Conference on Computer Vision and Pattern Recognition, 409-416.

Everingham, M. 2008. The pascal visual object classes challenge 2008 ( voc2008 ) development kit. International Journal of Computer Vision 111(1):98-136.

Fang, Y.; Chen, Z.; Lin, W.; and Lin, C. W. 2012. Saliency detection in the compressed domain for adaptive image retargeting. IEEE Transactions on Image Processing 21(9):3888-3901.

He, K.; Zhang, X.; Ren, S.; and Sun, J. 2016. Deep residual learning for image recognition. In Proc. of IEEE Conference on Computer Vision and Pattern Recognition, 770-778. 
Hou, Q.; Cheng, M.-M.; Hu, X.; Borji, A.; Tu, Z.; and Torr, P. 2017. Deeply supervised salient object detection with short connections. In Proc. of IEEE Conference on Computer Vision and Pattern Recognition, 5300-5309. IEEE.

Jiang, H.; Wang, J.; Yuan, Z.; Wu, Y.; Zheng, N.; and Li, S. 2013. Salient object detection: A discriminative regional feature integration approach. In Proc. of IEEE Conference on Computer Vision and Pattern Recognition, 2083-2090.

Krizhevsky, A.; Sutskever, I.; and Hinton, G. E. 2012. Imagenet classification with deep convolutional neural networks. In $\mathrm{Ad}$ vances in Neural Information Processing Systems, 1097-1105.

Lee, G.; Tai, Y. W.; and Kim, J. 2016. Deep saliency with encoded low level distance map and high level features. In Proc. of IEEE Conference on Computer Vision and Pattern Recognition, 660-668.

Li, G., and Yu, Y. 2015. Visual saliency based on multiscale deep features. In Proc. of IEEE Conference on Computer Vision and Pattern Recognition, 5455-5463.

Li, G., and Yu, Y. 2016. Deep contrast learning for salient object detection. In Proc. of IEEE Conference on Computer Vision and Pattern Recognition, 478-487.

Li, X.; Lu, H.; Zhang, L.; Xiang, R.; and Yang, M. H. 2013. Saliency detection via dense and sparse reconstruction. In Proc. of IEEE International Conference on Computer Vision, 29762983.

Li, Y.; Hou, X.; Koch, C.; Rehg, J. M.; and Yuille, A. L. 2014. The secrets of salient object segmentation. In Proc. of IEEE Conference on Computer Vision and Pattern Recognition, 280287.

Li, H.; Lu, H.; Lin, Z.; Shen, X.; and Price, B. 2015a. Lcnn: Low-level feature embedded cnn for salient object detection.

Li, X.; Zhao, L.; Wei, L.; Yang, M. H.; Wu, F.; Zhuang, Y.; Ling, H.; and Wang, J. 2015b. Deepsaliency: Multi-task deep neural network model for salient object detection. IEEE Transactions on Image Processing 25(8):3919.

Liu, N., and Han, J. 2016. Dhsnet: Deep hierarchical saliency network for salient object detection. In Proc. of IEEE Conference on Computer Vision and Pattern Recognition, 678-686.

Liu, T.; Yuan, Z.; Sun, J.; Wang, J.; Zheng, N.; Tang, X.; and Shum, H. Y. 2011. Learning to detect a salient object. IEEE Transactions on Pattern Analysis \& Machine Intelligence 33(2):353-367.

Liu, Y.; Jiang, P.-T.; Petrosyan, V.; Li, S.-J.; Bian, J.; Zhang, L.; and Cheng, M.-M. 2018. Del: Deep embedding learning for efficient image segmentation. In Proc. of International Joint Conference on Artificial Intelligence (IJCAI).

Luo, Z.; Mishra, A.; Achkar, A.; Eichel, J.; Li, S.; and Jodoin, P. M. 2017. Non-local deep features for salient object detection. In Proc. of IEEE Conference on Computer Vision and Pattern Recognition, 6593-6601.

Qin, Y.; Lu, H.; Xu, Y.; and Wang, H. 2015. Saliency detection via cellular automata. In Proc. of IEEE Conference on Computer Vision and Pattern Recognition, 110-119.

Schölkopf, B.; Platt, J.; and Hofmann, T. 2006. Graph-based visual saliency. In Advances in Neural Information Processing Systems, 545-552.

Schmid, C.; Jurie, F.; and Sharma, G. 2012. Discriminative spatial saliency for image classification. In Proc. of IEEE Conference on Computer Vision and Pattern Recognition, 3506-3513.
Wang, L.; Lu, H.; Xiang, R.; and Yang, M. H. 2015. Deep networks for saliency detection via local estimation and global search. In Proc. of IEEE Conference on Computer Vision and Pattern Recognition, 3183-3192.

Wang, L.; Wang, L.; Lu, H.; Zhang, P.; and Xiang, R. 2016. Saliency detection with recurrent fully convolutional networks. In Proc. of European Conference on Computer Vision, 825-841.

Wang, L.; Lu, H.; Wang, Y.; Feng, M.; Wang, D.; Yin, B.; and Ruan, X. 2017a. Learning to detect salient objects with imagelevel supervision. In Proc. of IEEE Conference on Computer Vision and Pattern Recognition, 3796-3805.

Wang, T.; Borji, A.; Zhang, L.; Zhang, P.; and Lu, H. 2017b. A stagewise refinement model for detecting salient objects in images. In Proc. of IEEE International Conference on Computer Vision, 4039-4048.

Wang, T.; Zhang, L.; Wang, S.; Lu, H.; Yang, G.; Ruan, X.; and Borji, A. 2018. Detect globally, refine locally: A novel approach to saliency detection. In Proc. of IEEE Conference on Computer Vision and Pattern Recognition, 3127-3135.

Wu, H.; Zheng, S.; Zhang, J.; and Huang, K. 2018. Fast endto-end trainable guided filter. In Proc. of IEEE Conference on Computer Vision and Pattern Recognition, 6450-6458.

Yan, Q.; Xu, L.; Shi, J.; and Jia, J. 2013. Hierarchical saliency detection. In Proc. of IEEE Conference on Computer Vision and Pattern Recognition, 1155-1162.

Yang, C.; Zhang, L.; Lu, H.; Xiang, R.; and Yang, M. H. 2013. Saliency detection via graph-based manifold ranking. In Proc. of IEEE Conference on Computer Vision and Pattern Recognition, 3166-3173.

Zeng, Y.; Lu, H.; Zhang, L.; Feng, M.; and Borji, A. 2018. Learning to promote saliency detectors. In Proc. of IEEE Conference on Computer Vision and Pattern Recognition, 38-56.

Zhang, P.; Wang, D.; Lu, H.; Wang, H.; and Ruan, X. 2017a. Amulet: Aggregating multi-level convolutional features for salient object detection. In Proc. of IEEE International Conference on Computer Vision, 202-211.

Zhang, P.; Wang, D.; Lu, H.; Wang, H.; and Yin, B. 2017 b. Learning uncertain convolutional features for accurate saliency detection. In Proc. of IEEE International Conference on Computer Vision, 212-221.

Zhang, L.; Dai, J.; Lu, H.; He, Y.; and Wang, G. 2018a. A bidirectional message passing model for salient object detection. In Proc. of IEEE Conference on Computer Vision and Pattern Recognition, 1741-1750.

Zhang, X.; Wang, T.; Qi, J.; Lu, H.; and Wang, G. 2018b. Progressive attention guided recurrent network for salient object detection. In Proc. of IEEE Conference on Computer Vision and Pattern Recognition, 248-255.

Zhang, L.; Shen, Y.; and Li, H. 2014. Vsi: A visual saliencyinduced index for perceptual image quality assessment. IEEE Transactions on Image Processing 23(10):4270-4281.

Zhao, R.; Ouyang, W.; Li, H.; and Wang, X. 2015. Saliency detection by multi-context deep learning. In Proc. of IEEE Conference on Computer Vision and Pattern Recognition, 1265-1274. 